\title{
THE MAXIMUM IDEMPOTENT-SEPARATING CONGRUENCE ON AN INVERSE SEMIGROUP
}

\author{
by J. M. HOWIE
}

(Received 31st December 1963)

A congruence $\rho$ on a semigroup will be called idempotent-separating if each $\rho$-class contains at most one idempotent. It is shown below that there exists a maximum such congruence $\mu$ on every inverse semigroup $S$. Two characterisations of $\mu$ are found, and it is shown (a) that $S / \mu \simeq E$, the semilattice of idempotents of $S$, if and only if $E$ is contained in the centre of $S$; (b) that $\mu$ is the identical congruence on $S$ if and only if $E$ is self-centralising, in a sense explained below.

A congruence $\rho$ on a semigroup $S$ is called a group congruence if $S / \rho$ is a group. It has been shown by Munn (4) that there exists a minimum such congruence $\sigma$ on every inverse semigroup. In Section 3 of this paper necessary and sufficient conditions are given for $\sigma \cap \mu$ to be the identical congruence and for $\sigma \vee \mu$ (the smallest congruence containing both $\sigma$ and $\mu$ ) to be the universal congruence.

\section{Definitions and preliminaries}

I shall use the terminology of Clifford and Preston (2). Two elements $a$ and $a^{\prime}$ of a semigroup will be called inverses of each other if

$$
a a^{\prime} a=a, \quad a^{\prime} a a^{\prime}=a^{\prime} .
$$

An inverse semigroup is a semigroup $S$ in which every element has a unique inverse. In such a semigroup, idempotent elements commute:

$$
e f=f e \text { if } e^{2}=e \text { and } f^{2}=f
$$

((2), Section 1.9). The unique inverse of the element $a$ is written $a^{-1}$. Then $a a^{-1}$ and $a^{-1} a$ are idempotents, and so also are $a e a^{-1}$ and $a^{-1} e a$, where $e$ is any idempotent in $S$. In fact $\alpha_{a}$, defined by

$$
e \alpha_{a}=a^{-1} e a,
$$

is a homomorphism of $E$, the subsemigroup of idempotents of $S$, into itself. We record also for future use that $e^{-1}=e$ if $e$ is idempotent, and that

$$
\left(a^{-1}\right)^{-1}=a, \quad(a b)^{-1}=b^{-1} a^{-1}
$$

for any $a, b$ in $S$. 
If $a$ and $b$ are two elements of an inverse semigroup $S$, we write $a \leqq b$ (or $b \geqq a$ ) if

$$
a a^{-1}=a b^{-1},
$$

or if any one of the following equivalent conditions holds:

$$
a a^{-1}=b a^{-1}, a^{-1} a=a^{-1} b, a^{-1} a=b^{-1} a .
$$

The relation $\leqq$ is a compatible order relation $(11,7)$ on $S$. The following observations - variously due to Vagner (10, 11), Preston (7) and Saĭn (9), and all readily verifiable - will be of use below. First,

$$
a^{-1} \leqq b^{-1} \text { if } a \leqq b .
$$

Also, if $e$ is any idempotent and if $a$ and $b$ are arbitrary elements of $S$, then

$$
e a \leqq a, \quad a e \leqq a, \quad a e b \leqq a b .
$$

The restriction of the order relation $\leqq$ to $E$, the subsemigroup of idempotents of $S$, is the natural semilattice order on $E$ : that is,

$$
e \leqq f \text { if and only if } e f=f e=e \text {. }
$$

Thus clearly $e f \leqq e$ and $e f \leqq f$ for any two idempotents $e, f$ in $S$.

If $H$ is an arbitrary subset of $S$, we denote by $H \omega$ the "closure" of $H$ with respect to the above order relation: that is,

$$
H \omega=\{a \in S: a \geqq h \text { for some } h \text { in } H\} .
$$

Ther $H \subseteq H \omega$ for any $H$. A subset $K$ will be called closed if $K \omega=K$. Clearly $H \omega$ is closed for any $H$.

If $E$ is the semilattice of idempotents of an inverse semigroup $S$, we define $E \zeta$, the centraliser of $E$ in $S$, by

$$
E \zeta=\{z \in S: e z=z e \text { for every } e \text { in } E\} .
$$

Clearly $E \subseteq E \zeta$. If $E \zeta=S$, then the idempotents are central, and the semigroup is a union of groups (1). If $E \zeta=E$, we shall say that $E$ is self-centralising. An example of an inverse semigroup whose semilattice of idempotents is self-centralising is the bicyclic semigroup ((2), Section 1.12).

A congruence $\rho$ on a semigroup $S$ is an equivalence relation which satisfies the condition that $a c \rho b c$ and $c a \rho c b$ for each $c$ in $S$ whenever $a \rho b$. If we denote the equivalence class containing $a$ by $a \rho$, then we can (not quite trivially) restate this condition as follows:

$$
(x \rho)(y \rho) \subseteq(x y) \rho
$$

for all $x, y \in S$. Thus $S / \rho$ can be given a semigroup structure in a natural way, and the mapping $\rho$ : $S \rightarrow S / \rho$ defined by

$$
x \rho \boxminus=x \rho
$$

is a homomorphism of $S$ onto $S / \rho$.

It is often convenient to consider a relation on $S$ as a subset of $S \times S$, and to write $(a, b) \in \rho$ rather than $a \rho b$. Thus, when $\rho$ and $\sigma$ are two congruences, 
the statement $\rho \subseteq \sigma$ and the expressions $\rho \cap \sigma, \rho \cup \sigma$ have the obvious set-theoretic meanings. It is easy to check that $\rho \cap \sigma$ is a congruence. On the other hand, $\rho \cup \sigma$ is not necessarily a congruence; we denote by $\rho \vee \sigma$ the smallest congruence containing $\rho$ and $\sigma$. We note that

$$
l_{S}=\{(x, x): x \in S\} \text { and } \omega_{s}=S \times S
$$

are congruences, which we call respectively the identical and the universal congruence on $S$.

If $\xi$ and $\eta$ are relations on a set $S$, then we write $\xi$ o $\eta$ for the relation consisting of all $(x, y)$ in $S \times S$ for which there exists $z$ in $S$ such that $(x, z) \in \xi$ and $(z, y) \in \eta$.

\section{The maximum idempotent-separating congruence}

As a starting point for our investigations we have the following theorem, and a lemma on which the theorem depends, both due to Vagner (10) and Preston (6).

Theorem 2.1. A homomorphic image of an inverse semigroup is an inverse semigroup.

Lemma 2.2. Let $\rho$ be a congruence on an inverse semigroup $S$. Then the inverse image $e(\rho t)^{-1}$ of an idempotent $e$ in $S / \rho$ contains an idempotent of $S$.

At this stage we record one consequence of the theorem which will be particularly useful.

Corollary 2.3. If $\rho$ is a congruence on an inverse semigroup, then $(x, y) \in \rho$ if and only if $\left(x^{-1}, y^{-1}\right) \in \rho$.

Proof. We denote the inverse semigroup by $S$. If $(x, y) \in \rho$, then the two elements $x \rho$ and $y \rho$ of $S / \rho$ are equal. It is easy to verify that $x^{-1} \rho$ and $y^{-1} \rho$ are both inverses of $x \rho$ in $S / \rho$, and so $x^{-1} \rho=y^{-1} \rho$ since $S / \rho$ is an inverse semigroup. That is $\left(x^{-1}, y^{-1}\right) \in \rho$. The converse follows from the fact that $\left(x^{-1}\right)^{-1}=x$ and $\left(y^{-1}\right)^{-1}=y$.

Theorem 2.4. Let $S$ be an inverse semigroup and let $\alpha_{a}$ be defined by (1) for any $a$ in $S$. Then the relation $\mu$ defined by the rule that $(x, y) \in \mu$ if and only if $\alpha_{x}=\alpha_{y}$ is the maximum idempotent-separating congruence on $S$.

Proof. It is immediate that $\mu$ is an equivalence relation. Now suppose that $(x, y) \in \mu$ and that $z$ is an arbitrary element of $S$. Then from the supposition that $x^{-1} e x=y^{-1} e y$ for every idempotent $e$ it follows immediately that $z^{-1} x^{-1} e x z=z^{-1} y^{-1} e y z$ for every idempotent $e$ : that is, $(x z, y z) \in \mu$. To show that $(z x, z y) \in \mu$, we note that $z^{-1} e z$ is an idempotent for every idempotent $e$, and so $x^{-1}\left(z^{-1} e z\right) x=y^{-1}\left(z^{-1} e z\right) y$ for every idempotent $e$. Thus $(z x, z y) \in \mu$ as required, and so $\mu$ is a congruence.

We next show that $\mu$ is idempotent-separating. Suppose that $(e, f) \in \mu$, where $e$ and $f$ are idempotents. Then, for every idempotent $g$, we have that $e^{-1} g e=f^{-1} g f:$ that is, $e g=f g$. The equality holds in particular when 
$g=e$; hence $e=f e$. We similarly obtain that $e f=f$ by putting $g=f$. Since $e f=f e$, it follows that $e=f$. Thus $\mu$ is idempotent-separating.

Finally, let $v$ be an idempotent-separating congruence on $S$; we shall show that $v \subseteq \mu$. Suppose that $(x, y) \in v$. Then $\left(x^{-1}, y^{-1}\right) \in v$ by Corollary 2.3 and, since $v$ is a congruence, it follows that $\left(x^{-1} e x, y^{-1} e y\right) \in v$ for every idempotent $e$. But both $x^{-1} e x$ and $y^{-1} e y$ are idempotents, and so it follows that $x^{-1} e x=y^{-1} e y$ for every idempotent $e$, since $v$ is by assumption idempotent separating. Thus $(x, y) \in \mu$ and so $\nu \subseteq \mu$ as required. This completes the proof of Theorem 2.4 .

An alternative characterisation of $\mu$ is provided by the next theorem.

Theorem 2.5. Let $S$ be an inverse semigroup with semilattice of idempotents $E$, let $\mu$ be the maximum idempotent-separating congruence on $S$, and let $E \zeta$ be the centraliser of $E$ in $S$. Then $(x, y) \in \mu$ if and only if $x^{-1} x=y^{-1} y$ and $x y^{-1} \in E \zeta$. Dually, $(x, y) \in \mu$ if and only if $x x^{-1}=y y^{-1}$ and $x^{-1} y \in E \zeta$.

Proof. It will be sufficient to prove the first of the two dual statements. Suppose first that $(x, y) \in \mu$, so that

$$
x^{-1} e x=y^{-1} e y
$$

for every $e$ in $E$. Then $\left(x^{-1}, y^{-1}\right) \in \mu$ by Corollary 2.3 ; that is, $x e x^{-1}=y e y^{-1}$ for every $e$ in $E$. Hence

$$
\begin{array}{r}
x^{-1} x=x^{-1} x \cdot x^{-1} x \cdot x^{-1} x=x^{-1} \cdot x\left(x^{-1} x\right) x^{-1} \cdot x=x^{-1} \cdot y\left(x^{-1} x\right) y^{-1} \cdot x \\
=y^{-1} \cdot y\left(x^{-1} x\right) y^{-1} \cdot y=y^{-1} y \cdot x^{-1} x \cdot y^{-1} y=x^{-1} x \cdot y^{-1} y
\end{array}
$$

and similarly $y^{-1} y=x^{-1} x \cdot y^{-1} y$. Thus $x^{-1} x=y^{-1} y$. Also, premultiplying both sides of (4) by $x$ and post-multiplying by $y^{-1}$, we have that

for every $e$ in $E$. Now,

$$
x x^{-1} e x y^{-1}=x y^{-1} e y y^{-1}
$$

and

$$
x x^{-1} e x y^{-1}=e x x^{-1} x y^{-1}=e x y^{-1}
$$

and so $e x y^{-1}=x y^{-1} e$ for every $e$ in $E$ : that is, $x y^{-1} \in E \zeta$.

Conversely, if $x^{-1} x=y^{-1} y$ and if $x y^{-1} \in E \zeta$, we have that $e x y^{-1}=x y^{-1} e$ for every $e$ in $E$. Premultiplying by $x^{-1}$ and postmultiplying by $y$, we obtain

$$
x^{-1} e x y^{-1} y=x^{-1} x y^{-1} e y \text {. }
$$

But $x^{-1} e x y^{-1} y=x^{-1} e x x^{-1} x=x^{-1} e x$, and similarly $x^{-1} x y^{-1} e y=y^{-1} e y$. Thus $x^{-1} e x=y^{-1} e y$ for every $e$ in $E$, and so $(x, y) \in \mu$ as required. This completes the proof.

Remark. It has been shown by Munn (5) that for inverse semigroups (and for certain other classes of regular semigroups) the idempotent-separating. congruences are precisely those contained in the equivalence relation $\mathscr{H}$ introduced by Green ((3); see also (2), Section 2.1), and that, in an arbitrary regular semigroup, the set of congruences contained in $\mathscr{H}$ forms a modular lattice with respect to the operations $\cap$ and $v$. 
Theorem 2.6. Let $S$ be an inverse semigroup with semilattice of idempotents $E$, and let $\mu$ be the maximum idempotent-separating congruence on $S$. Then $S / \mu \simeq E$ if and only if $E$ is central in $S$.

Proof. Since $\mu$ is idempotent-separating, it follows from Lemma 2.2 that $S / \mu$ is a semilattice if and only if each $\mu$-class contains exactly one idempotent. Thus, if $S / \mu$ is a semilattice, we must have that $S / \mu \simeq E$.

Suppose first that each $\mu$-class contains an idempotent. That is, for every $x$ in $S$ there exists an $f$ in $E$ such that $x^{-1} x=f^{-1} f$ and $x f^{-1} \in E \zeta$ (Theorem 2.5). Thus

$$
x=x x^{-1} x=x f^{-1} f=x f=x f^{-1} \in E \zeta .
$$

But this holds for any $x$ in $S$ and so $E \zeta=S$ as required.

Conversely, suppose that $E \zeta=S$. Then, in Theorem 2.5 , the condition that $x y^{-1} \in E \zeta$ becomes superfluous, and we have simply that $(x, y) \in \mu$ if and only if $x^{-1} x=y^{-1} y$. It is now clear that $\left(x, x^{-1} x\right) \in \mu$ for every $x$ in $S$, since $x^{-1} x=\left(x^{-1} x\right)^{-1}\left(x^{-1} x\right)$; hence every $\mu$-class contains an idempotent. This completes the proof.

Theorem 2.7. Let $S$ be an inverse semigroup with semilattice of idempotents $E$, and let $\mu$ be the maximum idempotent-separating congruence on $S$. Then $\mu=l_{S}$, the identical congruence on $S$, if and only if $E$ is self-centralising in $S$.

Proof. Suppose first that $\mu=t_{S}$, and let $z \in E \zeta$. Then, if we write $f$ for $z^{-1} z$, it is easy to see that $z^{-1} z=f^{-1} f(=f)$ and that $z f^{-1}(=z) \in E \zeta$. Thus $(z, f) \in \mu$ by Theorem 2.5 and so, since $\mu=t_{s}$, we have that $z=f \in E$. Thus $E \zeta=E$. 2.5 ,

Conversely, suppose that $E \zeta=E$, and let $(x, y) \in \mu$. Then, by Theorem

$$
x^{-1} x=y^{-1} y, \quad x x^{-1}=y y^{-1}, \text { and } x y^{-1}, \quad x^{-1} y \in E \zeta=E .
$$

Since the element $x y^{-1}$ is idempotent it must equal its inverse; i.e.

$$
x y^{-1}=y x^{-1} \text {. }
$$

Also, using the original characterisation of $\mu$ and the fact that $\left(x^{-1}, y^{-1}\right)$ belongs to $\mu$ if $(x, y)$ does, we have that

$$
\begin{aligned}
x x^{-1}=x x^{-1} \cdot x x^{-1}=x\left(x^{-1} x\right) x^{-1} & =y\left(x^{-1} x\right) y^{-1} \\
& =y x^{-1} \cdot x y^{-1}=\left(x y^{-1}\right)^{2}=x y^{-1} .
\end{aligned}
$$

Hence

$$
x=x x^{-1} x=x y^{-1} x=y x^{-1} x=y y^{-1} y=y \text {. }
$$

by (7), (6) and (5). Thus $\mu=l_{s}$, and the proof is complete.

If $S$ is an arbitrary inverse semigroup, then $S / \mu$ can have no non-identical idempotent-separating congruences, for if $v$ were such a congruence, then the relation $v^{\prime}$ on $S$ defined by the rule that $(x, y) \in v^{\prime}$ if and only if $(x \mu, y \mu) \in v$ would be an idempotent-separating congruence on $S$ properly containing $\mu-$ a contradiction. Hence we have the following corollary to Theorem 2.7: 
Corollary 2.8. Let $\mu$ be the maximum idempotent-separating congruence on an arbitrary inverse semigroup $S$. Then the semilattice of idempotents of $S / \mu$ is self-centralising.

Remark. It is easy to check that $\alpha_{x} \alpha_{y}=\alpha_{x y}$, so that the mapping $\alpha$ which sends $x$ to $\alpha_{x}$ is a representation. The homomorphism $\alpha_{x}$ can alternatively be considered as a partial one-to-one mapping of $E$ into itself thus: $\alpha_{x}$ maps $\left\{e \in E: e \leqq x x^{-1}\right\}$ in a one-to-one manner onto $\left\{e \in E: e \leqq x^{-1} x\right\}$. Considered in this way, the representation becomes identical to that described by Preston in ((7), Section 3). The condition for $\alpha$ to be faithful given by Theorem 2.7 above appears to be new. We also remark that the partial one-to-one mappings $\alpha_{x}$ are restrictions to $E$ of the partial isomorphisms considered by Preston in (8).

\section{The minimum group congruence}

For an arbitrary inverse semigroup $S$, Munn (4) has given the following characterisation of $\sigma$, the minimum group congruence: $(x, y) \in \sigma$ if and only if there exists an idempotent $e$ in $S$ such that $e x=e y$. An alternative characterisation is provided by the next theorem.

Theorem 3.1. Let $S$ be an inverse semigroup with semilattice of idempotents $E$, and let $\sigma$ be the minimum group congruence on $S$. Then $(x, y) \in \sigma$ if and only if $x y^{-1} \in E \omega$.

Proof. Suppose first that $e x=e y$ for some $e$ in $E$. Then $e x y^{-1}=e y y^{-1} \in E$. Now $x y^{-1} \geqq e x y^{-1}$ by (3), and so $x y^{-1} \in E \omega$.

Conversely, suppose that $x y^{-1} \in E \omega$. Then there exists $f$ in $E$ such that $x y^{-1} \geqq f$, i.e. such that $f x y^{-1}=f$. If we write $e$ for $f x y^{-1} y x^{-1}$, then $e \in E$ and clearly $e f=e$. Also,

$$
e x=e^{2} x=e f x y^{-1} y x^{-1} x=e f x x^{-1} x y^{-1} y=e f x y^{-1} y=e f y=e y .
$$

Thus Theorem 3.1 is proved.

This characterisation of $\sigma$ is the key to the proof of the next theorem.

Theorem 3.2. Let $\sigma$ be the minimum group congruence and $\mu$ the maximum idempotent-separating congruence on an inverse semigroup $S$ with semilattice of idempotents $E$. Then $\sigma \cap \mu=l_{S}$ if and only if $E \omega \cap E \zeta=E$.

Proof. By Theorems 2.5 and 3.1, we have that $(x, y) \in \sigma \cap \mu$ if and only if $x^{-1} x=y^{-1} y$ and $x y^{-1} \in E \omega \cap E \zeta$. Suppose first that $E \omega \cap E \zeta=E$ and that $(x, y) \in \sigma \cap \mu$. The equalities (6), (7) and (8) then follow exactly as in the proof of Theorem 2.7. Thus $x=y$, and so $\sigma \cap \mu=l_{s}$ as required.

Conversely, suppose that $\sigma \cap \mu=\imath_{S}$, and let $z \in E \omega \cap E \zeta$. If we denote $z^{-1} z$ by $e$, it is clear that $z e^{-1}(=z e=z)$ belongs to $E \omega$; hence $(z, e) \in \sigma$. Also, $z e^{-1}=z \in E \zeta$ and $z^{-1} z=e^{-1} e(=e)$; hence $(z, e) \in \mu$ (Theorem 2.5). Since by assumption $\sigma \cap \mu=\imath_{s}$, we must therefore have that $z=e \in E$. Hence $E \omega \cap E \zeta=E$ as required. This completes the proof. 
We require some preliminaries before investigating the nature of $\sigma \vee \mu$. A subsemigroup $H$ of an inverse semigroup $S$ is called an inverse subsemigroup if $x^{-1}$ belongs to $H$ whenever $x$ does. An inverse subsemigroup $H$ of $S$ will be called self-conjugate if $z x z^{-1}$ belongs to $H$ for any $z$ whenever $x$ belongs to $H$.

The next two lemmas are implicit in Šain's paper (9).

Lemma 3.3. Let $K$ be a closed, self-conjugate inverse subsemigroup of an inverse semigroup $S$. Suppose further that $K \supseteq E$, the semilattice of idempotents of $S$. Then the relation $\rho_{K}$ defined by the rule that $(x, y) \in \rho_{K}$ if and only if $x y^{-1} \in K$ is a congruence on $S$.

Proof. Since $x x^{-1} \in E \subseteq K$, we have that $\rho_{K}$ is reflexive. It is symmetric since $y x^{-1}=\left(x y^{-1}\right)^{-1}$ belongs to $K$ whenever $x y^{-1}$ does. Suppose now that $x y^{-1}, y z^{-1} \in K$. Then $x y^{-1} y z^{-1} \in K$ since $K$ is a subsemigroup. But

$$
x z^{-1} \geqq x y^{-1} y z^{-1}
$$

by (3), and so $x z^{-1} \in K$ since $K$ is closed. Thus $\rho_{K}$ is transitive.

Now suppose that $x y^{-1} \in K$ and that $z$ is an arbitrary element of $S$. Then $(z x)(z y)^{-1}=z x y^{-1} z^{-1} \in K$ since $K$ is self-conjugate. Also,

$$
(x z)(y z)^{-1}=x z z^{-1} y^{-1}=x y^{-1} \cdot y z z^{-1} y^{-1} \in K . E \subseteq K .
$$

Thus $\rho_{K}$ is a congruence.

Lemma 3.4. If $H$ is a self-conjugate inverse subsemigroup of an inverse semigroup $S$, then so is $H \omega$.

Proof. Let $x$ and $y$ be elements of $H \omega$, and let $h$ and $k$ be the elements of $H$ such that $x \geqq h$ and $y \geqq k$. From the compatibility of the order relation it now follows that $x y \geqq h k \in H$; hence $x y \in H \omega$. By (2), we have that

$$
x^{-1} \geqq h^{-1} \in H
$$

hence $x^{-1} \in H \omega$. Thus $H \omega$ is an inverse subsemigroup. Finally, if $z$ is an arbitrary element of $S$, it follows, again from the compatibility of the order relation, that $z x z^{-1} \geqq z h z^{-1} \in H$; hence $z x z^{-1} \in H \omega$.

We also have .

Lemma 3.5. Let $S$ be an inverse semigroup with semilattice of idempotents $E$. Then the centraliser $E \zeta$ of $E$ in $S$ is a self-conjugate inverse subsemigroup of $S$.

Proof. It is clear that $x y$ belongs to $E \zeta$ if $x$ and $y$ do. If $x \in E \zeta$, then $x e=e x$ for every $e$ in $E$. Taking inverses, we find that $e x^{-1}=x^{-1} e$ for every $e$ in $E$; hence $x^{-1} \in E \zeta$. Now let $x \in E \zeta$ and let $z$ be an arbitrary element of $S$. Then

$$
\begin{aligned}
z x z^{-1} e=z x z^{-1} z z^{-1} e & =z x z^{-1} e z z^{-1}=z\left(x \cdot z^{-1} e z\right) z^{-1} \\
= & z\left(z^{-1} e z \cdot x\right) z^{-1}=z z^{-1} e z x z^{-1}=e z z^{-1} z x z^{-1}=e z x z^{-1}
\end{aligned}
$$

for every $e$ in $E$; hence $z x z^{-1} \in E \zeta$.

As an immediate consequence of the last two lemmas, we have 
Lemma 3.6. If $S$ is an inverse semigroup with semilattice of idempotents $E$, then $(E \zeta) \omega$ is a closed, self-conjugate inverse subsemigroup of $S$.

Since $(E \zeta) \omega$ certainly contains $E$, it now follows from Lemma 3.3 that the relation $\rho_{(E \zeta) \omega}$, which from now on we shall denote simply by $\rho$, is a congruence on $S$.

The following theorem characterises $\sigma \vee \mu$.

Theorem 3.7. Let $\sigma$ be the minimum group congruence and $\mu$ the maximum idempotent-separating congruence on an inverse semigroup $S$ with semilattice of idempotents $E$. Then the relation $\rho$, defined by the rule that $(x, y) \in \rho$ if and only if $x y^{-1} \in(E \zeta) \omega$ is equal to $\sigma \vee \mu$.

Proof. We have already remarked that $\rho$ is a congruence on $S$. Moreover, it follows immediately from Theorems 3.1 and 2.5 that $\sigma \subseteq \rho$ and $\mu \subseteq \rho$; hence $\sigma \vee \mu \subseteq \rho$. It remains to prove that $\rho \subseteq \sigma \vee \mu$. We prove in fact that $\rho \subseteq \sigma \circ \mu \circ \sigma$, which is clearly sufficient.

Suppose, then, that $(x, y) \in \rho$. Then there exists $z \in E \zeta$ such that $x y^{-1} \geqq z$. Let

$$
u=z y \text { and } v=z^{-1} z y .
$$

Then $x u^{-1}=x y^{-1} z^{-1} \geqq z z^{-1} \in E$ and so $x u^{-1} \in E \omega$. Thus $(x, u) \in \sigma$. Also,

$$
v^{-1} v=y^{-1} z^{-1} z z^{-1} z y=y^{-1} z^{-1} z y=u^{-1} u
$$

and, for every $e$ in $E$,

$$
\begin{aligned}
u v^{-1} e & \left.=z y y^{-1} z^{-1} z e=z e y y^{-1} z^{-1} z \quad \text { (since } y y^{-1} z^{-1} z \in E\right) \\
& \left.=e z y y^{-1} z^{-1} z=e u v^{-1} \quad \text { (since } z \in E \zeta\right) .
\end{aligned}
$$

Thus $u v^{-1} \in E \zeta$ and so, by Theorem 2.5 , we have that $(u, v) \in \mu$. Finally, $v y^{-1}=z^{-1} z y y^{-1} \in E \subseteq E \omega$, and so $(v, y) \in \sigma$. Summarising, we have that

$$
(x, u) \in \sigma, \quad(u, v) \in \mu, \quad(v, y) \in \sigma,
$$

and so $(x, y) \in \sigma \circ \mu \circ \sigma$ as required. This completes the proof.

An obvious consequence of the theorem is

Corollary 3.8. The smallest congruence $\sigma \vee \mu$ containing $\sigma$ and $\mu$ is the universal congruence if and only if $(E \zeta) \omega=S$.

Proof. It is clear that $\sigma \vee \mu=\omega_{S}$ if $(E \zeta) \omega=S$. Conversely, if $\sigma \vee \mu=\omega_{S}$, then $x y^{-1} \in(E \zeta) \omega$ for all $x, y$ in $S$. In particular, for all $x$ in $S$,

Thus $(E \zeta) \omega=S$.

$$
x\left(x^{-1} x\right)^{-1}=x x^{-1} x=x \in(E \zeta) \omega .
$$

Note. In the proof of Theorem 3.7 it emerged incidentally that

$$
\sigma \vee \mu=\sigma \circ \mu \circ \sigma \text {. }
$$

This remains true if $\mu$ is replaced by any congruence whatever on $S$ :

Theorem 3.9. Let $\sigma$ be the minimum group congruence on an inverse semigroup $S$, and let $\xi$ be an arbitrary congruence on $S$. Then $\sigma \vee \xi=\sigma \circ \xi \circ \sigma$. 
Proof. Clearly $\sigma \circ \xi \circ \sigma \subseteq \sigma \vee \xi$. To show the opposite inclusion it suffices to prove that $\sigma \circ \xi \circ \sigma$ is transitive, for it is then a congruence containing $\sigma$ and $\xi$ (and therefore containing $\sigma \vee \xi$ ). Suppose, then, that $(x, y)$ and $(y, z)$ belong to $\sigma \circ \xi \circ \sigma$. Then there exist $a, b, c, d$ in $S$ such that

$$
\begin{array}{lll}
(x, a) \in \sigma, & (a, b) \in \xi, & (b, y) \in \sigma, \\
(y, c) \in \sigma, & (c, d) \in \xi, & (d, z) \in \sigma .
\end{array}
$$

Now, by the transitivity of $\sigma$, we have immediately that $(b, c) \in \sigma$, and so there exists an idempotent $e$ such that $e b=e c$. By the left-compatibility of $\xi$, we have that

$$
(e a, e b) \in \xi, \quad(e c, e d) \in \xi,
$$

and so $(e a, e d) \in \xi$. Moreover, $e a=e . e a$, and so $(a, e a) \in \sigma$; hence, by transitivity, $(x, e a) \in \sigma$. A similar argument shows that $(e d, z) \in \sigma$. Hence, summarising, we have that

$$
(x, e a) \in \sigma, \quad(e a, e d) \in \xi, \quad(e d, z) \in \sigma,
$$

and so $(x, z) \in \sigma \circ \xi \circ \sigma$ as required.

\section{REFERENCES}

(1) A. H. Clifford, Semigroups admitting relative inverses, Annals of Math., 42 (1941), 1037-1049.

(2) A. H. Clifford and G. B. Preston, The Algebraic Theory of Semigroups, vol. 1, Math. Surveys of the American Math. Soc. 7 (Providence, R.I., 1961).

(3) J. A. Green, On the structure of semigroups, Annals of Math., 54 (1951), 163-172.

(4) W. D. MUNN, A class of irreducible matrix representations of an arbitrary inverse semigroup, Proc. Glasgow Math. Assoc., 5 (1961), 41-48.

(5) W. D. MunN, A certain sublattice of the lattice of congruences on a regular semigroup, Proc. Cambridge Phil. Soc. (to appear).

(6) G. B. Preston, Inverse semi-groups, J. London Math. Soc.; 29 (1954), 396-403.

(7) G. B. Preston, Representations of inverse semi-groups, J. London Math. Soc., 29 (1954), 411-419.

(8) G. B. Preston, A note on representations of inverse semigroups, Proc. American Math. Soc., 8 (1957), 1144-1147.

(9) B. M. SAIN, Representations of generalised groups, Izvestiya Vysšikh Učebnykh Zavedenil (Matematika), 28 (1962), 164-176 (Russian).

(10) V. V. VAGNeR, The theory of generalised groups and generalised heaps, Mat. Sbornik (N.S.), 32 (1953), 545-632 (Russian).

(11) V. V. VAGner, Generalised groups, Doklady Akad. Nauk SSSR, 84 (1952), 1119-1122 (Russian).

\section{Department of Mathematics}

THE UNIVERSITY

GlasGow, W.2 